\title{
Presencia de Caulerpaceae (Chlorophyta) en la península DE YuCATÁN, MÉXICO
}

\author{
Julio Espinoza-Avalos ${ }^{1}$, luis E. Aguilar-Rosas², Raúl Aguilar-Rosas 3 , \\ Jorge M. GÓmez-PoOt ${ }^{4}$ y Rodolfo Raigoza-Figueras ${ }^{5}$ \\ ${ }^{1}$ El Colegio de la Frontera Sur, Chetumal, Quintana Roo, México \\ 2Instituto de Investigaciones Oceanológicas, Universidad Autónoma de Baja California, \\ Ensenada, Baja California, México \\ ${ }^{3}$ Facultad de Ciencias Marinas, Universidad Autónoma de Baja California, Ensenada, Baja California, México \\ ${ }^{4}$ Comisión Nacional de Áreas Naturales Protegidas, Secretaría de Medio Ambiente y Recursos Naturales Chetumal, \\ Quintana Roo, México \\ ${ }^{5}$ Acuario Xcaret, Playa de Carmen, Quintana Roo, México \\ ${ }^{1}$ Autor para la correspondencia: jespino@ecosur.mx
}

\begin{abstract}
Resumen: Veintitrés taxones de Caulerpaceae (14 especies, 3 variedades y 6 formas) se encontraron en 70 sitios a lo largo de las costas de la península de Yucatán, México. La macroalga invasora $C$. brachypus f. parvifolia se reporta por primera vez en Quintana Roo. El análisis cualitativo de clasificación jerárquica aglomerativa ("clustering") mostró ausencia de agrupamientos de sitios representativos de las costas del Caribe mexicano y del Golfo de México, en contraste con otros estudios que han reportado diferencias a ambos lados de la península de Yucatán, con base a la presencia de otros grupos de la biota marina. Caulerpa cupressoides, $C$. prolifera y $C$. verticillata fueron las especies con más registros (en 30, 23 y 21 sitios, respectivamente), mientras que $C$. brachypus, C. lanuginosa, C. microphysa y C. webbiana fueron las menos comunes, con un registro cada especie.
\end{abstract}

Palabras clave: Caulerpa, Caribe mexicano, Golfo de México, macroalgas invasoras, Quintana Roo.

\begin{abstract}
Twenty-three taxa of Caulerpaceae (14 species, 3 varieties, and 6 forms) were found in 70 sites along the coast of the Yucatan peninsula, Mexico. The invasive macroalgae C. brachypus f. parvifolia is reported for the first time in Quintana Roo, Mexico. A hierarchical agglomerative clustering analysis showed the nonexistence of sites grouping at both sides of the Yucatan peninsula, in contrast with other studies that have reported differences at both sides of the peninsula based on the presence of other groups of the marine biota. Caulerpa cupressoides, C. prolifera, and C. verticillata were the species having more records (in 30, 23, and 21 sites, respectively), while C. brachypus, C. lanuginosa, C. microphysa, and C. webbiana were among the less common species, with one record each species.
\end{abstract}

Key words: Caulerpa, Gulf of Mexico, Mexican Caribbean, invasive macroalgae, Quintana Roo.

$\mathbf{L}$ a familia Caulerpaceae incluye a algas verdes cenocíticas y comprende a los géneros Caulerpa y Caulerpella, siendo el primero más diverso en especies, con respecto al último que sólo contiene una especie (Prud'Homme et al., 1996). Caulerpa es uno de los géneros predominantes y de amplia distribución en mares tropicales y subtropicales del mundo, con algunas especies presentes en aguas templadas (Carruthers et al., 1993). Hasta finales de la década de los 80`s, el número de especies de Caulerpa reconocido consistía de aproximadamente 75 taxones (Price et al., 1998).
Actualmente, el número de especies aceptadas taxonómicamente de este género es 93 (Guiry y Guiry, 2014).

El Mar Caribe, la región de Malesia y la parte sur de Australia, representan centros con alta diversidad de especies de Caulerpa (Prud'Homme et al., 1996). En algunas partes del Caribe, la presencia de algunas especies de Caulerpa ha sido evaluada particularmente abundante (De-Rios, 1972; John y Price, 1979; Norris y Bucher, 1982). En muchos estudios florísticos de la península de Yucatán se han incluido especies del género Caulerpa, no obstante, en estudios re- 
cientes siguen reportándose nuevos registros de especies de Caulerpa para los tres estados que conforman la península de Yucatán (Campeche, Yucatán y Quintana Roo) (Mendoza-González et al., 2000; Robledo y Freile-Pelegrín, 2005; Ortegón-Aznar et al., 2008; Pacheco-Cervera et al., 2010).

Al delimitar la distribución mundial de organismos marinos, la punta noreste de la península de Yucatán se ha señalado como el límite entre ecorregiones (Caribe Occidental y Sur del Golfo de México; Spalding et al., 2007) y regiones (Golfo de México y Caribe; Costello et al., 2010). Particularmente, esta punta se ha considerado como la frontera o zona de transición entre zonas biogeográficas del Mar Caribe y el Golfo de México. En lo que respecta a la fauna, Díaz-Merlano y Puyana-Hegedus (1994) y Salazar-Vallejo (2000) separaron las subprovincias Macroantillana y del Golfo de México. De manera similar, para el caso de macroalgas marinas, Díaz-Martín y Espinoza-Avalos (2000) y Aguilar-Rosas et al. (2001) consideraron que la punta noreste de la península indicaba una frontera en la distribución de taxones de Phaeophyceae, Halimedaceae y Udoteaceae.
El objetivo de este estudio fue determinar la presencia de taxones Caulerpaceae a lo largo de las costas de la península de Yucatán, y si la punta noreste de la Península delimitaba asociaciones de especies de Caulerpaceae, comparando la estructura de la comunidad con base en la presencia o ausencia de los taxones de Caulerpaceae entre el Golfo de México y el Caribe mexicano.

\section{Materiales y métodos}

La península de Yucatán se localiza en la parte sureste de México e incluye los estados de Campeche y Yucatán hacia el Golfo de México y Quintana Roo hacia el Mar Caribe (Figura 1).

Múltiples ejemplares de Caulerpaceae se recolectaron de ambientes marinos en diferentes épocas del año, de septiembre de 1991 a abril de 2001, sin seguir un patrón de muestreo. Los ejemplares se separaron de muestras generales de macroalgas y pastos marinos. Con la excepción de Xcaret, el muestreo de ejemplares no se repitió en ninguno de los 70
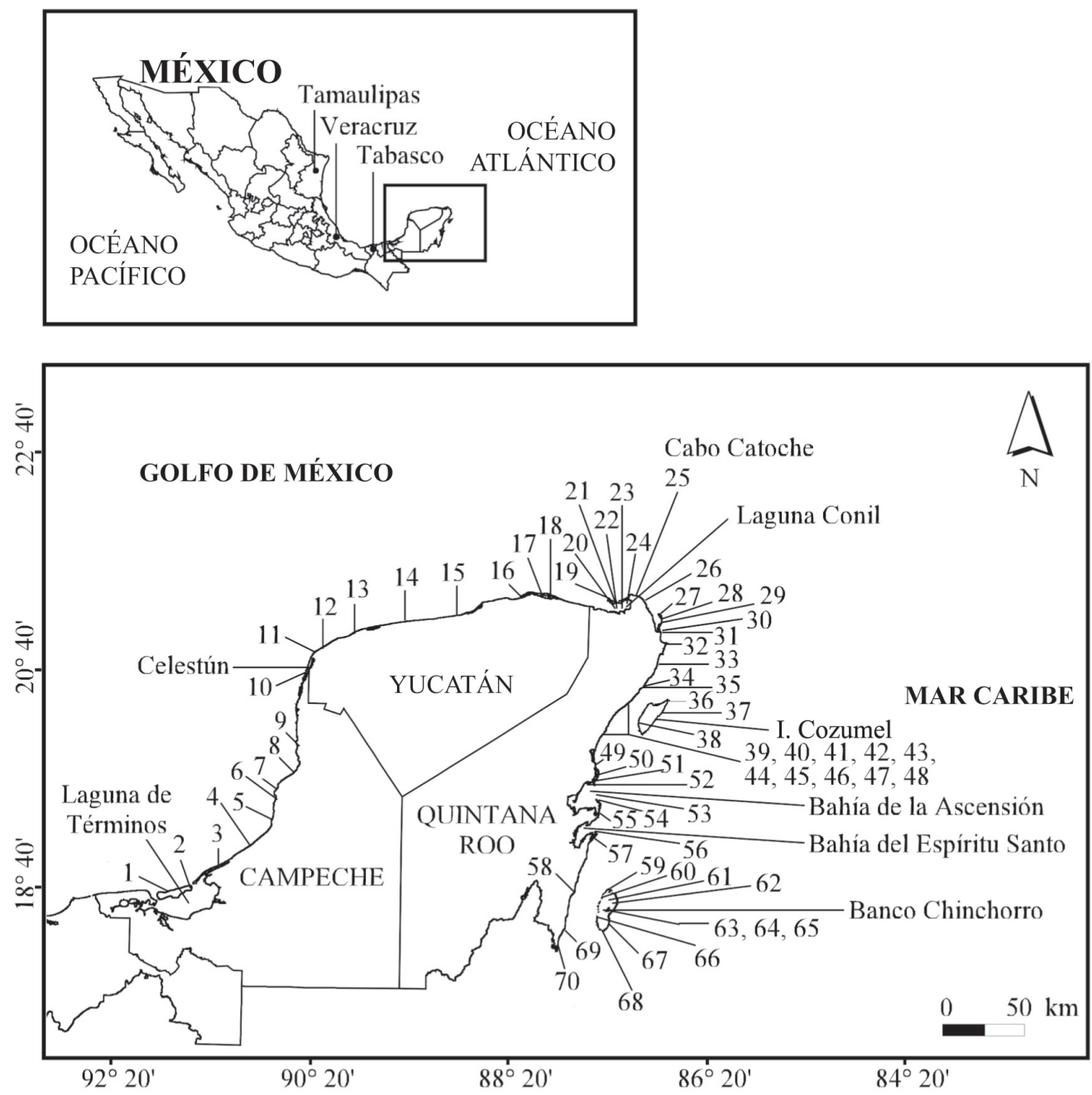

Figura 1. Península de Yucatán, mostrando los sitios donde se recolectaron los especímenes de Caulerpaceae. 
sitios que se incluyen en este estudio. El nombre, la fecha de recolecta y la posición geográfica de los sitios de muestreo se muestran en la tabla 1. Las muestras se recolectaron aleatoriamente y sin un procedimiento con fines cuantitativos. Algunas muestras se obtuvieron de caletas y cenotes. Las algas se recolectaron manualmente mediante buceo libre y autónomo, usualmente en sitios de menos de $4 \mathrm{~m}$ de profundidad. Las algas se preservaron en formalina al $4 \%$ diluida con agua de mar. La salinidad se midió con un refractómetro manual $\mathrm{ATAGO}^{\circledR} \mathrm{S} / \mathrm{Mill}$, únicamente en caletas y cenotes.

Las determinaciones taxonómicas se llevaron a cabo usan- do microscopios estereoscópico Olympus y fotónico Carl Zeiss con iluminación de campo claro, siguiendo los trabajos de Weber-van-Bosse (1898), Taylor (1960) y Dawes y Mathieson (2008). La nomenclatura de las especies se basó en Wynne (2011), y las actualizaciones nomenclaturales se obtuvieron de AlgaeBase (Guiry y Guiry, 2014).

Los especímenes recolectados se depositaron en el Herbario de El Colegio de la Frontera Sur, Unidad Chetumal, México (= CIQR), en dos modos de preservación: secos en papel de herbario y en formalina al $4 \%$.

Para determinar la existencia de posibles diferencias en

Tabla 1. Nombre, posición geográfica y fecha de colecta de los sitios donde se encontraron especies Caulerpaceae en la península de Yucatán. Los números de sitios corresponden con los de la figura 1 .

\begin{tabular}{|c|c|c|c|c|c|c|c|c|c|}
\hline \multicolumn{2}{|c|}{ Sitio Nombre } & \multirow[t]{2}{*}{$\begin{array}{l}\text { Posición } \\
\text { Latitud N }\end{array}$} & Longitud O & \multirow[t]{2}{*}{$\begin{array}{l}\text { Fecha } \\
\text { de colecta }\end{array}$} & \multicolumn{2}{|c|}{ Sitio Nombre } & \multirow[t]{2}{*}{$\begin{array}{l}\text { Posición } \\
\text { Latitud N }\end{array}$} & \multirow[t]{2}{*}{ Longitud O } & \multirow[t]{2}{*}{$\begin{array}{l}\text { Fecha } \\
\text { de colecta }\end{array}$} \\
\hline$\overline{\text { Cam }}$ & mpeche & & & & 4 & Roo & & & \\
\hline 1 & Punta Bajamita & $18^{\circ} 43^{\prime} 38.30^{\prime \prime}$ & $91^{\circ} 38^{\prime} 58.73^{\prime \prime}$ & 15-11-1993 & 35 & Punta Maroma & $20^{\circ} 43^{\prime} 42.41^{\prime \prime}$ & $86^{\circ} 57^{\prime} 48.54^{\prime \prime}$ & $19-0$ \\
\hline 2 & Puerto Real & & $11^{\prime \prime}$ & 13-11-1993 & 36 & Punta Molas & $20^{\circ} 35^{\prime} 0$ & $86^{\circ}$ & $07-($ \\
\hline 3 & Sabancuy & $19^{\circ} 01^{\prime} 19.15^{\prime \prime}$ & $91^{\circ} 12^{\prime} 43.06^{\prime \prime}$ & 13-11-1993 & 37 & Playa Chen Río & $20^{\circ} 22^{\prime} 55.57^{\prime \prime}$ & $86^{\circ} 52$ & $09-0$ \\
\hline 4 & Chenkán & $19^{\circ} 08^{\prime} 47.37^{\prime \prime}$ & $90^{\circ} 57^{\prime} 26.04^{\prime \prime}$ & 12-11-1993 & 38 & Playa San Francisco & $20^{\circ} 22^{\prime} 04.64^{\prime \prime}$ & $87^{\circ} 01^{\prime} 31.60^{\prime \prime}$ & 07-04-1992 \\
\hline 5 & Tide pools & $19^{\circ} 16^{\prime} 24.14^{\prime \prime}$ & $90^{\circ} 47^{\prime} 39.55^{\prime \prime}$ & 16-11-1993 & 39 & Xcaret & $20^{\circ} 34^{\prime} 04.25^{\prime \prime}$ & $87^{\circ} 06^{\prime} 36.09^{\prime \prime}$ & -2001 \\
\hline 6 & Punta Morro & ${ }^{\circ} 40^{\prime} 30.24 \prime$ & $0^{\circ} 42^{\prime} 25.21^{\prime \prime}$ & 12-11-1993 & 40 & Puerto Av & $20^{\circ} 29^{\prime} 53.12^{\prime \prime}$ & $87^{\circ} 13^{\prime} 37.46^{\prime \prime}$ & $26-0$ \\
\hline 7 & Playa Bonita & $9^{\circ} 47^{\prime} 40.62^{\prime \prime}$ & $0^{\circ} 37^{\prime} 17.46^{\prime \prime}$ & 11-11-1993 & 41 & Xpu-há & $20^{\circ} 28^{\prime} 48.24^{\prime \prime}$ & $9.01^{\prime \prime}$ & $26-$ \\
\hline 8 & Ría San Francisco & $19^{\circ} 51^{\prime} 47.62^{\prime \prime}$ & $90^{\circ} 31^{\prime} 54.78^{\prime \prime}$ & 11-11-1993 & 42 & Yalkú & $20^{\circ} 24^{\prime} 43.24^{\prime \prime}$ & $87^{\circ} 18^{\prime}$ & $25-05$ \\
\hline 9 & Tenabo & $20^{\circ} 05^{\prime} 41.65^{\prime \prime}$ & $90^{\circ} 28^{\prime} 52.75^{\prime \prime}$ & 11-11-1993 & 43 & Akumal & $20^{\circ} 23^{\prime} 43.25^{\prime \prime}$ & $87^{\circ} 18^{\prime} 49.97^{\prime \prime}$ & 12-03-1992 \\
\hline \multicolumn{2}{|c|}{ Yucatán } & & & & 44 & & $20^{\circ} 20^{\prime} 24.03^{\prime \prime}$ & $87^{\circ} 20^{\prime} 33.75^{\prime \prime}$ & 993 \\
\hline 10 & Celestún & $20^{\circ} 52^{\prime} 31.47^{\prime \prime}$ & $90^{\circ} 23^{\prime} 43.81^{\prime \prime}$ & $11-08$ & 45 & Xel-há & $20^{\circ} 19^{\prime} 04.09^{\prime \prime}$ & $87^{\circ} 21^{\prime} 27.91^{\prime \prime}$ & 24-05-1995 \\
\hline 11 & Punta Piedra & $21^{\circ} 09^{\prime} 22.65^{\prime \prime}$ & $90^{\circ} 05^{\prime} 46.18^{\prime \prime}$ & 12-08-1995 & 46 & Predio San Francisco & $20^{\circ} 17^{\prime} 18.17^{\prime \prime}$ & $87^{\circ} 22^{\prime} 30.00^{\prime \prime}$ & $24-C$ \\
\hline 12 & Sisal & $21^{\circ} 09^{\prime} 56.59^{\prime \prime}$ & $90^{\circ} 02^{\prime} 57.44^{\prime \prime}$ & 12-08-1995 & 47 & Tankah & $20^{\circ} 15^{\prime} 59.34^{\prime \prime}$ & $87^{\circ} 23^{\prime} 28.97^{\prime \prime}$ & $23-0$ \\
\hline 13 & Progreso & $21^{\circ} 18^{\prime} 33.25^{\prime \prime}$ & $89^{\circ} 40^{\prime} 22.07^{\prime \prime}$ & 12-08-1995 & 48 & Shangri-La & $20^{\circ} 05^{\prime} 27.96^{\prime \prime}$ & $87^{\circ} 28$ & $12-0$ \\
\hline 14 & Telchac P & $21^{\circ} 20^{\prime} 43.48^{\prime \prime}$ & $89^{\circ} 15^{\prime} 35.24^{\prime \prime}$ & & 49 & & $19^{\circ} !$ & $87^{\circ} 2$ & \\
\hline 15 & Dzilam de Bravo & $21^{\circ} 23^{\prime} 45.76^{\prime \prime}$ & $88^{\circ} 52^{\prime} 50.72^{\prime \prime}$ & 13-08-1995 & 50 & Punta Xoquem & $19^{\circ} 49^{\prime} 58.12^{\prime \prime}$ & $87^{\circ} 27^{\prime} 16.71^{\prime \prime}$ & 07-10-1991 \\
\hline 16 & San Felipe & $21^{\circ} 34^{\prime} 07.63^{\prime \prime}$ & $88^{\circ} 14^{\prime} 13.87^{\prime \prime}$ & 13-08-1995 & 51 & Vigía Chico & $19^{\circ} 46^{\prime} 24.69^{\prime \prime}$ & $87^{\circ} 35^{\prime} 09.75^{\prime \prime}$ & 08-10-1991 \\
\hline 17 & Las Coloradas & $.52^{\prime \prime}$ & $87^{\circ} 58^{\prime} 54.06^{\prime \prime}$ & $14-08$ & 52 & Cayo Culebras & $19^{\circ} 42^{\prime} 19.38^{\prime \prime}$ & $87^{\circ} 29^{\prime} 48.08^{\prime \prime}$ & 1991 \\
\hline 18 & El Cuyo & $31^{\prime \prime}$ & $87^{\circ} 44^{\prime} 31.02^{\prime \prime}$ & $14-0$ & 53 & Arrecife del Barco & $19^{\circ} 44^{\prime} 20.12^{\prime \prime}$ & $87^{\circ} 25^{\prime} 12.86^{\prime \prime}$ & 06- \\
\hline \multicolumn{2}{|c|}{ Quintana Roo } & & & & 54 & Punta Piedra & $19^{\circ} 33^{\prime} 29.91^{\prime \prime}$ & $87^{\circ} 24^{\prime} 54.95^{\prime \prime}$ & 07-10-1991 \\
\hline 19 & Punta Sirin & $21^{\circ} 30^{\prime} 10.64^{\prime \prime}$ & $87^{\circ} 23^{\prime} 26.57^{\prime \prime}$ & 09-09-1993 & 55 & La Victoria & $19^{\circ} 26^{\prime} 39.16^{\prime \prime}$ & $87^{\circ} 28^{\prime} 11.87^{\prime \prime}$ & 24-09-1991 \\
\hline 20 & Chiquilá & $21^{\circ} 26^{\prime} 00.56^{\prime \prime}$ & $87^{\circ} 20^{\prime} 10.91^{\prime \prime}$ & 08-09-1993 & 56 & Candelaria de Chal & $19^{\circ} 21^{\prime} 36.15^{\prime \prime}$ & $87^{\circ} 29^{\prime} 54.44^{\prime \prime}$ & 26-09-1991 \\
\hline 21 & Punta Mosquito & $21^{\circ} 33^{\prime} 45.00^{\prime \prime}$ & $87^{\circ} 19^{\prime} 48.44^{\prime \prime}$ & 07-09-1993 & 57 & La Sardina & $19^{\circ} 15^{\prime} 36.64^{\prime \prime}$ & $87^{\circ} 29^{\prime} 15.93^{\prime \prime}$ & 1991 \\
\hline 22 & Punta Chen & $21^{\circ} 26^{\prime} 28.60^{\prime \prime}$ & $87^{\circ} 19^{\prime} 02.10^{\prime \prime}$ & 09-09-1993 & 58 & Río Indio & $18^{\circ} 45^{\prime} 44.18^{\prime \prime}$ & $87^{\circ} 40^{\prime} 06.57^{\prime \prime}$ & 28-02-1992 \\
\hline 23 & Punta Chuchú & $21^{\circ} 25^{\prime} 47.08^{\prime \prime}$ & $87^{\circ} 13^{\prime} 36.36^{\prime \prime}$ & 08-09-1993 & 59 & Cayo Norte & $18^{\circ} 45^{\prime} 04.47^{\prime \prime}$ & $87^{\circ} 18^{\prime} 17.96^{\prime \prime}$ & 20-10-1991 \\
\hline 24 & Bajo Media Luna & $21^{\circ} 27^{\prime} 13.46^{\prime \prime}$ & $87^{\circ} 09^{\prime} 54.83^{\prime \prime}$ & 08-09-1993 & 60 & Bajo & $18^{\circ} 42^{\prime} 51.83^{\prime \prime}$ & $87^{\circ} 21^{\prime} 57.72^{\prime \prime}$ & 18-10-1991 \\
\hline 25 & Cabo Catoche & $21^{\circ} 36^{\prime} 18.66^{\prime \prime}$ & $87^{\circ} 06^{\prime} 14.68^{\prime \prime}$ & 07-09-1993 & 61 & Agua Verde & $18^{\circ} 36^{\prime} 28.95^{\prime \prime}$ & $87^{\circ} 16^{\prime} 51.45^{\prime \prime}$ & 19-10-1991 \\
\hline 26 & Boca Iglesias & $21^{\circ} 31^{\prime} 51.03^{\prime \prime}$ & $86^{\circ} 57^{\prime} 47.29^{\prime \prime}$ & 07-09-1993 & 62 & San Andrés & $18^{\circ} 33^{\prime} 39.00^{\prime \prime}$ & $87^{\circ} 17^{\prime} 45.31^{\prime \prime}$ & 20-10-1991 \\
\hline 27 & Bahía Chacmochuc & $21^{\circ} 25^{\prime} 42.91^{\prime \prime}$ & $86^{\circ} 50^{\prime} 39.00^{\prime \prime}$ & 16-03-1992 & 63 & Cayo Centro NE & $18^{\circ} 35^{\prime} 55.73^{\prime \prime}$ & $87^{\circ} 18^{\prime} 12.39^{\prime \prime}$ & 20-10-1991 \\
\hline 28 & Isla Blanca & $21^{\circ} 25^{\prime} 53.92^{\prime \prime}$ & $86^{\circ} 48^{\prime} 27.51^{\prime \prime}$ & 16-03-1992 & 64 & Cayo Centro SW & $18^{\circ} 34^{\prime} 50.00^{\prime \prime}$ & $87^{\circ} 20^{\prime} 05.75^{\prime \prime}$ & 18-10-1991 \\
\hline 29 & Punta Sam & $21^{\circ} 13^{\prime} 58.90^{\prime \prime}$ & $86^{\circ} 48^{\prime} 03.41^{\prime \prime}$ & 15-03-1992 & 65 & La Herradura & $18^{\circ} 30^{\prime} 33.88^{\prime \prime}$ & $87^{\circ} 18^{\prime} 47.94^{\prime \prime}$ & 18-10-1991 \\
\hline 30 & Isla Mujeres & $21^{\circ} 15^{\prime} 20.28^{\prime \prime}$ & $86^{\circ} 44^{\prime} 54.09^{\prime \prime}$ & 01-06-1993 & 66 & Cordillera & $18^{\circ} 29^{\prime} 22.64^{\prime \prime}$ & $87^{\circ} 25^{\prime} 47.48^{\prime \prime}$ & 19-10-1991 \\
\hline 31 & Bajo Pepito & $21^{\circ} 13^{\prime} 09.23^{\prime \prime}$ & $86^{\circ} 44^{\prime} 15.23^{\prime \prime}$ & 14-04-1994 & 67 & El Tropic & $18^{\circ} 29^{\prime} 20.21^{\prime \prime}$ & $87^{\circ} 18^{\prime} 41.51^{\prime \prime}$ & 19-10-1991 \\
\hline 32 & Laguna Bojórquez & $21^{\circ} 05^{\prime} 20.85^{\prime \prime}$ & $86^{\circ} 46^{\prime} 24.30^{\prime \prime}$ & 05-06-1996 & 68 & Cayo Lobos & $18^{\circ} 23^{\prime} 41.17^{\prime \prime}$ & $87^{\circ} 23^{\prime} 22.42^{\prime \prime}$ & 19-10-1991 \\
\hline 33 & Punta Caracol & $20^{\circ} 53^{\prime} 20.32^{\prime \prime}$ & $86^{\circ} 51^{\prime} 38.17^{\prime \prime}$ & 19-05-1992 & 69 & Punta Xcayal & $18^{\circ} 23^{\prime} 15.12^{\prime \prime}$ & $87^{\circ} 46^{\prime} 58.13^{\prime \prime}$ & 27-02-1992 \\
\hline 34 & La Caleta & $20^{\circ} 47^{\prime} 02.57^{\prime \prime}$ & $86^{\circ} 56^{\prime} 15.85^{\prime \prime}$ & 19-05-1992 & 70 & Xcalak & $18^{\circ} 16^{\prime} 28.65^{\prime \prime}$ & $87^{\circ} 50^{\prime} 03.29^{\prime \prime}$ & 08-11-1997 \\
\hline
\end{tabular}


la estructura de la comunidad de las Caulerpaceae recolectadas en aguas del Golfo de México y del Caribe mexicano, se utilizó la técnica de clasificación jerárquica aglomerativa ("clustering"). Con los datos de presencia-ausencia de los taxones de Caulerpa se elaboró una matriz de similitud de distancias de Bray-Curtis, utilizando el método de ligamiento promedio. Para evaluar la evidencia genuina de agrupaciones de los sitios de estudio se usó la prueba SIMPROF (prueba de permutaciones). Los análisis se realizaron con el software Primer 6 (Clarke y Gorley, 2006). Para contrarrestar el menor número de sitios visitados en Campeche y Yucatán con respecto a los de Quintana Roo, en los análisis cualitativos se agregaron los registros de Caulerpa en sitios reportados por otros autores para los dos estados del Golfo de México.

\section{Resultados}

Veintitrés taxones de Caulerpaceae (14 especies, 3 variedades y 6 formas) se encontraron en 70 sitios de la península de Yucatán, México (Tabla 2). Caulerpa cupressoides, $C$. prolifera, $C$. verticillata y $C$. sertularioides fueron las especies con mayor presencia en la península, (en 30, 23 y 21 sitios, respectivamente), mientras que $C$. brachypus, $C$. lanuginosa, $C$. microphysa, $C$. webbiana y Caulerpella ambigua fueron las especies menos comunes, con un registro las cuatro primeras y dos registros la última especie (Tabla 2).
Caulerpa verticillata estuvo presente en las caletas de Yalkú, Predio San Francisco y Xel-há (con salinidad de 25 PSU), al igual que $C$. sertularioides en las caletas de los sitios 42, 45, 46, y 47 (Figura 1). Caulerpa fastigiata fue la única especie que se recolectó en cenotes, como Xpu-há y Tankah, donde los valores de salinidad fueron 15 PSU y 12 PSU, respectivamente.

Un piso completamente cubierto por especies de Cauler$p a$, principalmente $C$. prolifera, se observó en rocas de Punta Bajamita, Campeche (15-11-1993) y en fondos lodosos de Dzilám de Bravo, Yucatán (13-07-1995). Otras especies de Caulerpa se encontraban mezcladas con $C$. prolifera en los sitios 1 y 15 (Figura 1).

Caulerpa brachypus f. parvifolia (Harvey) A. B. Cribb es un nuevo registro para el Caribe mexicano, se recolectó el 04-04-2001 y el 11-04-2001 a $24 \mathrm{~m}$ y $27 \mathrm{~m}$ de profundidad, respectivamente, enfrente del Parque Xcaret (sitio 39, Figura 1). Los ejemplares se encontraban creciendo encima de un conglomerado compuesto de escombro de corales, arena y la esponja Geodia sp. Céspedes de C. brachypus f. parvifolia se trasplantaron a los tanques de exhibición del parque. Estas algas (frondas con estípite corto, planas, $3(-4) \mathrm{cm}$ de largo, sin ramificarse, ovales a elongadas, con márgenes lisos, raramente dentados), aparentemente muy resistentes, agresivamente colonizaron el sustrato ocupado por otros organismos que se encontraban en los tanques de exhibición. Para controlar su capacidad de invasión, los dos primeros

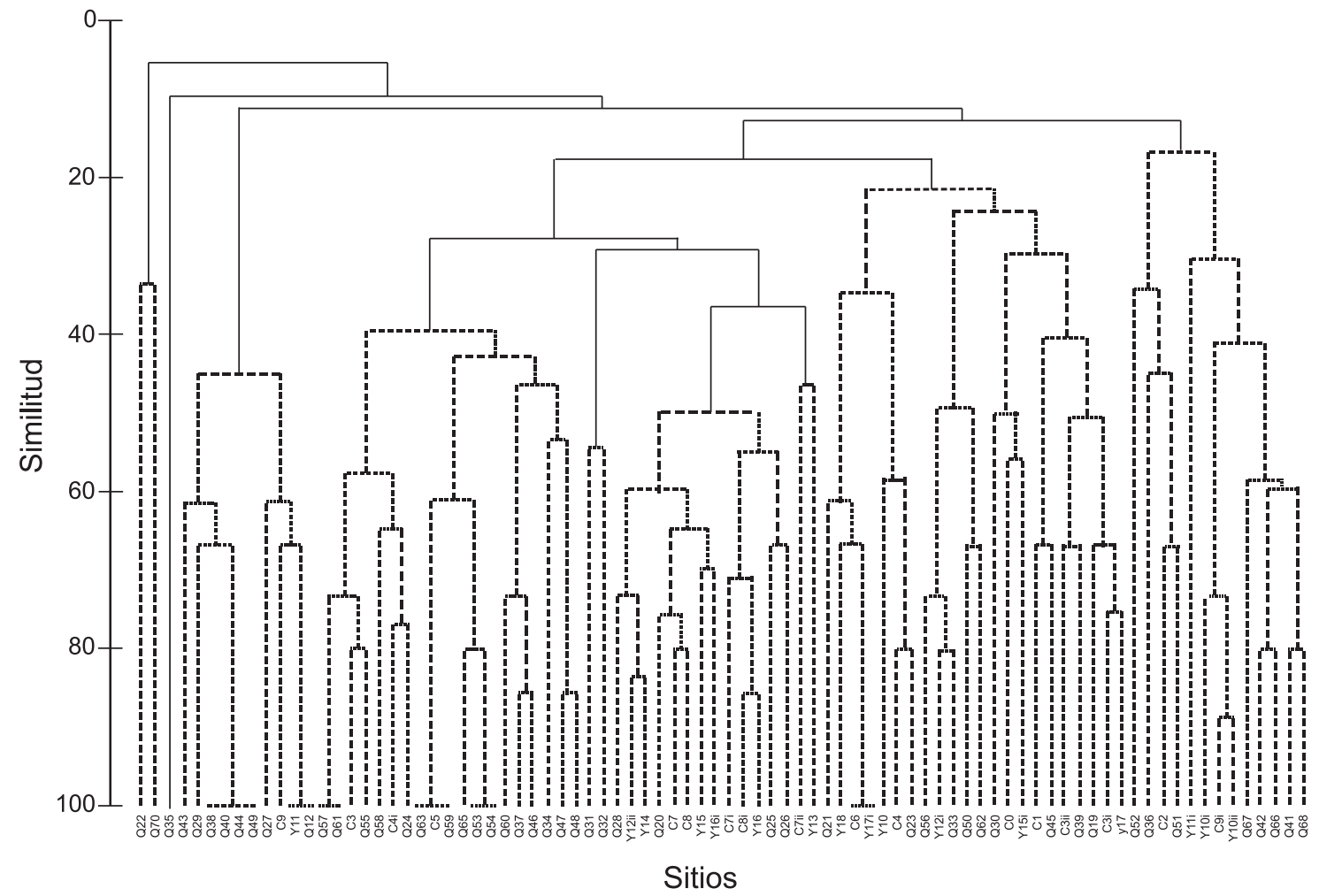

Figura 2. Dendrograma de agrupamientos por sitios de recolecta, con base a datos de presencia-ausencia; C (Campeche), Q (Quintana Roo) y Y (Yucatán), en la península de Yucatán. 
años fue necesario podarlas aproximadamente cada mes, hasta que alcanzaron una condición de crecimiento estable, y finalmente desaparecieron después de cinco años (2006) de estar en exhibición. En la naturaleza, C. brachypus f. parvifolia desapareció después del paso por la zona de Xcaret de los huracanes Emily y Wilma, el 18-07-2005 y 21-102005, ambos con categoría 4 de la escala Saffir-Simpson. El paso de estos huracanes causó modificaciones extremas a los hábitats de arrecife de coral a 15-27 m de profundidad, y posteriormente $C$. brachypus f. parvifolia no se ha observado en los alrededores de Xcaret.

El número total de especies de Caulerpaceae para la península de Yucatán, cuando se sumaron los taxones encontrados en este estudio y los reportados en la literatura por otros autores, fue 36 (15 especies, 11 variedades y 10 formas), como se muestra en la tabla 3. Las especies con mayor presencia en la península fueron $C$. racemosa, $C$. prolifera, C. cupressoides y C. mexicana (en 26, 25, 24 y 24 sitios, respetivamente), mientras que las menos comunes fueron $C$. serrulata, C. brachypus, C. lanuginosa y C. webbiana (en 1, 2, 5 y 6 sitios, respectivamente; Tabla 3).

El análisis de agrupamiento mostró que la comunidad de Caulerpa fue similar a uno y otro lado de la península de Yucatán, sugiriendo la ausencia de grupos bien definidos que permitan separar las comunidades de ambos lados de la península (Figura 2). La prueba SIMPROF indicó que las agrupaciones sugeridas por el dendrograma únicamente son significativas a valores de similitud menores a $29.1 \%(\mathrm{Pi}=$

Tabla 2. Taxones Caulerpaceae encontrados en cada estado en la península de Yucatán. Los números representan los sitios de colecta indicados en la figura 1.

\begin{tabular}{|c|c|c|c|c|c|c|c|}
\hline TAXÓN & CAMPECHE & $\begin{array}{l}\text { ESTADO } \\
\text { YUCATÁN }\end{array}$ & $\begin{array}{l}\text { QUINTANA } \\
\text { ROO }\end{array}$ & TAXÓN & CAMPECHE & $\begin{array}{l}\text { ESTADO } \\
\text { YUCATÁN }\end{array}$ & $\begin{array}{l}\text { QUINTANA } \\
\text { ROO }\end{array}$ \\
\hline $\begin{array}{l}\text { C. brachypus f. parvifolia } \\
\text { (Harvey) A.B.Cribb }\end{array}$ & 8 & $\begin{array}{l}13,14,15 \\
16\end{array}$ & $\begin{array}{l}20,24,25, \\
26,28 \\
14\end{array}$ & C. peltata J.V.Lamouroux & 2 & 15,17 & $\begin{array}{l}30,36,42, \\
51,52,53 \\
54,65,66 \\
68\end{array}$ \\
\hline $\begin{array}{l}\text { C. cupressoides (West in } \\
\text { Vahl) C.Agardh }\end{array}$ & $3,5,7,8$ & $14,15,16$ & $\begin{array}{l}20,24,25, \\
28,32,34, \\
35,37,46, \\
47,48,53, \\
54,55,56, \\
57,58,59, \\
60,61,63, \\
65,66\end{array}$ & $\begin{array}{l}\text { f. obovata J.Agardh } \\
\text { f. zosterifolia Børgesen }\end{array}$ & $\begin{array}{l}4,7,8 \\
1\end{array}$ & $\begin{array}{l}14,15,16 \\
17,18\end{array}$ & $\begin{array}{l}19,20,21, \\
23,24,25, \\
28,30,31, \\
32,33,55, \\
56 \\
22,23,24\end{array}$ \\
\hline $\begin{array}{l}\text { var. mamillosa (Montagne) } \\
\text { Weber-van Bosse } \\
\text { C. fastigiata Montagne }\end{array}$ & 1 & 15,17 & $19,39,45$ & C. racemosa (Forsskål) J.Agardh & 7,8 & $13,16,17$ & $\begin{array}{l}20,24,30 \\
34,41,42 \\
47,48,58 \\
66,67,68\end{array}$ \\
\hline C. lanuginosa J.Agardh & & & 30 & $\begin{array}{l}\text { C. sertularioides (S.G.Gmelin) } \\
\text { M.A.Howe }\end{array}$ & 1,3 & 14,15 & $\begin{array}{l}23,24,28 \\
29,30,38\end{array}$ \\
\hline C. mexicana Sonder ex Kützing & 1 & 15 & $\begin{array}{l}19,24,30 \\
33,37,50 \\
56,57,58 \\
61,62\end{array}$ & & & & $\begin{array}{l}40,43,44, \\
45,49,55, \\
57,61\end{array}$ \\
\hline $\begin{array}{l}\text { f. laxior (Weber-van Bosse) } \\
\text { W.R.Taylor }\end{array}$ & & & 50 & $\begin{array}{l}\text { f. farlowii (Weber-van Bosse) } \\
\text { Børgesen }\end{array}$ & & & $\begin{array}{l}37,46,47, \\
52,60\end{array}$ \\
\hline $\begin{array}{l}\text { C. microphysa (Weber-van } \\
\text { Bosse) Feldmann }\end{array}$ & & & 36 & $\begin{array}{l}\text { f. longiseta (Bory de Saint- } \\
\text { Vincent) Svedelius }\end{array}$ & & & 31 \\
\hline $\begin{array}{l}\text { C. paspaloides (Bory de } \\
\text { Saint-Vincent) Greville }\end{array}$ & & & $\begin{array}{l}19,20,22 \\
25,26,34 \\
37,46\end{array}$ & C. verticillata J.Agardh & $7,8,9$ & $\begin{array}{l}11,12,14 \\
15,17,18\end{array}$ & $\begin{array}{l}19,20,27 \\
31,34,38 \\
40,43,44 \\
49,52,65\end{array}$ \\
\hline $\begin{array}{l}\text { var. compressa (Weber-van } \\
\text { Bosse) M.A. Howe }\end{array}$ & & & $\begin{array}{l}20,24,28 \\
30,32,35 \\
47,48\end{array}$ & $\begin{array}{l}\text { C. webbiana Montagne } \\
\text { Caulerpella ambigua (Okamura) } \\
\text { Prud'homme et Lokhorst }\end{array}$ & & & $\begin{array}{l}30 \\
27,36\end{array}$ \\
\hline $\begin{array}{l}\text { var. wurdemanni Weber-van } \\
\text { Bosse }\end{array}$ & 8,9 & 15 & 21 & & & & \\
\hline
\end{tabular}




\section{Julio Espinoza-Avalos ET AL.}

Tabla 3. Taxones Caulerpaceae registrados en este y otros estudios en cada estado de la península de Yucatán. Los números representan las referencias de los registros.

\begin{tabular}{|c|c|c|c|c|c|c|c|}
\hline TAXÓN & CAMPECHE & $\begin{array}{l}\text { ESTADO } \\
\text { YUCATÁN }\end{array}$ & $\begin{array}{l}\text { QUINTANA } \\
\text { ROO }\end{array}$ & TAXÓN & CAMPECHE & $\begin{array}{l}\text { ESTADO } \\
\text { YUCATÁN }\end{array}$ & $\begin{array}{l}\text { QUINTANA } \\
\text { ROO }\end{array}$ \\
\hline C. ashmeadii Harvey & 25,32 & $\begin{array}{l}5,11 \mathrm{i}, 12, \\
16,20 \mathrm{ii}, \\
22,29,31 \mathrm{i}, \\
32\end{array}$ & $\begin{array}{l}9,16,19, \\
32\end{array}$ & C. peltata J.V.Lamouroux & $\begin{array}{l}16,17,21^{\mathrm{e}}, \\
32\end{array}$ & $\begin{array}{l}1 \mathrm{e}, 16,22 \\
32\end{array}$ & $\begin{array}{l}1^{\mathrm{e}}, 9,12, \\
13^{\mathrm{f}}, 16,16^{\mathrm{f}} \\
19^{\mathrm{e}}, 20^{\mathrm{e}} \\
27,32\end{array}$ \\
\hline $\begin{array}{l}\text { C. brachypus f. parvifolia } \\
\text { (Harvey) A.B.Cribb }\end{array}$ & & 23 & 32 & $\begin{array}{l}\text { C. prolifera (Forsskål) } \\
\text { J.V.Lamouroux }\end{array}$ & $\begin{array}{l}6,16,17 \\
21,25,32\end{array}$ & $\begin{array}{l}11 \mathrm{i}, 16 \\
20 \mathrm{ii}, 22 \\
24,29\end{array}$ & $\begin{array}{l}1,8,9,12 \\
13,14,16 \\
19,20 \mathrm{i}\end{array}$ \\
\hline $\begin{array}{l}\text { C. cupressoides (West) } \\
\text { C.Agardh }\end{array}$ & 6,32 & $\begin{array}{l}11 \mathrm{i}, 12, \\
20 \mathrm{ii}, 22,24 \\
29,31 \mathrm{i}, 32\end{array}$ & $\begin{array}{l}1,8,9,12 \\
13,16,18 \\
19,20 \\
20 i, 27,28 \\
31,32\end{array}$ & $\begin{array}{l}\text { f. obovata J. Agardh } \\
\text { f. zosterifolia Børgesen }\end{array}$ & $\begin{array}{l}15,16,32 \\
21,32\end{array}$ & $\begin{array}{l}31 \mathrm{i}, 32 \\
16,22,32 \\
16\end{array}$ & $\begin{array}{l}27,32 \\
1,9,16,32 \\
16\end{array}$ \\
\hline $\begin{array}{l}\text { var. lycopodium Weber- } \\
\text { van Bosse }\end{array}$ & 25 & $16^{\mathrm{a}}$ & $\begin{array}{l}9,10,27 \\
30 \\
10,27,30^{\mathrm{a}}\end{array}$ & C. racemosa (Forsskål) J.Agardh & $\begin{array}{l}6,14^{\mathrm{h}}, 16^{\mathrm{h}} \\
25,32\end{array}$ & $\begin{array}{l}11 \mathrm{i}, 14^{\mathrm{h}} \\
16^{\mathrm{h}}, 20 \mathrm{ii} \\
24,29,32\end{array}$ & $\begin{array}{l}1,2,5,8 \\
9,13,14^{\mathrm{h}} \\
16^{\mathrm{h}}, 19,20 \\
27,28,31 \\
32\end{array}$ \\
\hline $\begin{array}{l}\text { var. mamillosa (Montagne) } \\
\text { Weber-van Bosse }\end{array}$ & 16 & 16 & $\begin{array}{l}1,2,13 \\
16,27,32\end{array}$ & $\begin{array}{l}\text { var. macrophysa (Sonder ex } \\
\text { Kützing) W.R.Taylor }\end{array}$ & 16 & 16 & $\begin{array}{l}11,13,16 \\
20\end{array}$ \\
\hline $\begin{array}{l}\text { var. serrata (Kützing) } \\
\text { Weber-van Bosse }\end{array}$ & 16 & & & $\begin{array}{l}\text { var. occidentalis (J.Agardh) } \\
\text { Børgesen }\end{array}$ & 21 & 16 & 13,16 \\
\hline var. turneri Weber-van Bosse & 15,16 & 16 & $\begin{array}{l}1,2,13 \\
16,20\end{array}$ & $\begin{array}{l}\text { var. turbinata (J.Agardh) Eubank } \\
\text { C. serrulata (Forsskål) J.Agardh }\end{array}$ & & & $\begin{array}{l}16^{g} \\
20\end{array}$ \\
\hline C. fastigiata Montagne & $\begin{array}{l}15^{\mathrm{b}}, 16, \\
21^{\mathrm{b}}, 32\end{array}$ & $16,22,32$ & $\begin{array}{l}7,10,13 \\
16,27,28 \\
30,32\end{array}$ & $\begin{array}{l}\text { C. sertularioides (S.G.Gmelin) } \\
\text { M.A.Howe }\end{array}$ & $6,16,32$ & $\begin{array}{l}16,20 \mathrm{ii}, \\
24,32\end{array}$ & $\begin{array}{l}1,2,9,11 \\
12,16,19 \\
20,20 \mathrm{i} 27\end{array}$ \\
\hline C. lanuginosa J.Agardh & & & $\begin{array}{l}3,8,19 \\
27,32\end{array}$ & & & & $\begin{array}{l}28,30,31 i, \\
32\end{array}$ \\
\hline C. mexicana Sonder ex Kützing & $6,25,32$ & $\begin{array}{l}4,11 \mathrm{i}, 16 \\
16^{c}, 29,32\end{array}$ & $\begin{array}{l}1,4,8,9 \\
13,16,16^{c} \\
18,19,20 \\
27,28,30 \\
31 \mathrm{i}, 32\end{array}$ & $\begin{array}{l}\text { f. brevipes (J.Agardh) Svedelius } \\
\text { f. corymbosa W.R.Taylor }\end{array}$ & 14,21 & 16 & $\begin{array}{l}1,9,13 \\
16,19,20 i \\
27\end{array}$ \\
\hline $\begin{array}{l}\text { f. laxior (Weber-van Bosse) } \\
\text { W.R.Taylor }\end{array}$ & & & $4,16,32$ & $\begin{array}{l}\text { f. farlowii (Weber-van Bosse) } \\
\text { Børgesen }\end{array}$ & & 16 & $\begin{array}{l}1,13,14 \\
16,19,27 \\
32\end{array}$ \\
\hline f. pectinata (Kützing) Taylor & 21 & & 4,20 & $\begin{array}{l}\text { f. longiseta (Bory de Saint- } \\
\text { Vincent) Svedelius }\end{array}$ & 16,21 & 16,22 & $\begin{array}{l}1,9,13 \\
16,32\end{array}$ \\
\hline $\begin{array}{l}\text { C. microphysa (Weber-van } \\
\text { Bosse) Feldmann }\end{array}$ & $17^{\mathrm{d}}$ & & $\begin{array}{l}1,8,13 \\
20,20 i, 27 \\
32\end{array}$ & C. verticillata J. Agardh & 16,32 & $\begin{array}{l}16,22,23 \\
24,32\end{array}$ & $\begin{array}{l}1,5,8,9 \\
11,12,16 \\
19,20,20 i\end{array}$ \\
\hline $\begin{array}{l}\text { C. paspaloides (Bory de Saint- } \\
\text { Vincent) Greville }\end{array}$ & 25 & $11 \mathrm{i}, 29,31 \mathrm{i}$ & $\begin{array}{l}1,2,8,9 \\
12,13,19 \\
20,20 \mathrm{i}, 27\end{array}$ & & & & $\begin{array}{l}27,28,30, \\
32\end{array}$ \\
\hline & & & 28,32 & C. webbiana Montagne & & 23 & $\begin{array}{l}20,26,27 \\
28,32\end{array}$ \\
\hline $\begin{array}{l}\text { var. compressa (Weber-van } \\
\text { Bosse) M.A.Howe }\end{array}$ & & 20ii & $13,27,32$ & $\begin{array}{l}\text { Caulerpella ambigua (Okamura) } \\
\text { Prud'homme \& Lokhorst }\end{array}$ & $14^{k}, 16^{j}$ & $16^{i}, 23$ & $\begin{array}{l}1^{\mathrm{k}}, 13^{\mathrm{j}}, 16^{\mathrm{i}} \\
19^{\mathrm{k}}, 20,27^{\prime}\end{array}$ \\
\hline var. laxa Weber-van Bosse & & & 16 & & & & 28,32 \\
\hline $\begin{array}{l}\text { f. phleoides (Bory de Saint- } \\
\text { Vincent) Weber-van Bosse }\end{array}$ & & & 16 & \multirow{2}{*}{\multicolumn{4}{|c|}{$\begin{array}{l}\text { REFERENCIAS: 1) Aguilar-Rosas et al., 1998; 2) Aguilar-Rosas et al., } \\
\text { 1989; 3) Aguilar-Rosas et al., 1992; 4) Apartado et al., 2002; 5) Bas- } \\
\text { tida-Zavala et al.,2000; 6) Callejas-Jiménez et al., 2005; 7) Collado- } \\
\text { Vides et al., 1995; 8) Collado-Vides et al., 1998; 9) Díaz-Martín et al., } \\
\text { 1998; 10) Díaz-Martín y Quan-Young, 2001; 11) Espinoza-Avalos et } \\
\text { al., 2009; 11i) Freile-Pelegrín y Morales, 2004; 12) Garduño-Solórzano }\end{array}$}} \\
\hline $\begin{array}{l}\text { var. wurdemanni Weber- } \\
\text { van Bosse }\end{array}$ & 16,32 & 16,32 & $16,27,32$ & & & & \\
\hline
\end{tabular}


Tabla 3. Continuación.

et al., 2005; 13) Garza-Barrientos, 1975; 14) Huerta-Múzquiz, 1958; 15) Huerta-Múzquiz y Garza-Barrientos, 1966; 16) Huerta-Múzquiz et al., 1987; 17) Humm, 1952; 18) Mateo-Cid et al., 2006; 19) Mendoza-González y Mateo-Cid, 1992; 20) Mendoza-González et al., 2000; 20i) Mendoza-González et al., 2007; 20ii) Okolodkov et al., 2014; 21) Ortega, 1995; 22) Ortegón-Aznar et al., 2001; 23) Ortegón-Aznar et al., 2008; 24) Ortegón-Aznar et al., 2009; 25) Pacheco-Cervera et al., 2010; 26) Quan-Young et al., 1998; 27) Quan-Young et al., 2004; 28) Quan-Young et al., 2006; 29) Robledo y Freile-Pelegrín, 2005; 30) Serviere-Zaragoza et al., 1992; 31) Tussenbroek y Collado-Vides, 2000; 31i) Zubia et al., 2007; 32) Este estudio. ${ }^{a}$ como C. cupressoides var. lycopodium (J. Agardh) Weber-van Bosse, ${ }^{\mathrm{b}}$ como C. fastigiata var. confervoides $\mathrm{P}$. et $\mathrm{H}$. Crouan ex Weber-van Bosse, ${ }^{\mathrm{c}}$ como C. taxifolia (Vahl) C. Agardh (ver Apartado et al. 2002), ' como C. racemosa var. microphysa (Weber-van Bosse) Taylor, ${ }^{\text {e }}$ como C. racemosa var. peltata (Lamouroux) Eubank, ${ }^{\mathrm{f}}$ como Caulerpa racemosa var. laetevirens (Montagne) Weber-van Bosse, ${ }^{g}$ como C. racemosa var. chemnitzia (Esper) Weber-van Bosse, ${ }^{\mathrm{h}}$ como C. racemosa var. uvifera (Turner) J. Agardh, 'i como C. racemosa var. clavifera (Turner) Weber-van Bosse, j como C. vickersiae var. luxurians Taylor, ${ }^{k}$ como C. vickersiae Børgesen, ' como Caulerpa ambigua Okamura.

4.51; $P<0.1 \%)$. A este nivel de similitud se formaron siete grupos; sin embargo, estos están compuestos por muestras de ambos lados de la península de Yucatán.

\section{Discusión}

El número relativamente alto de las especies Caulerpaceae registradas para la península de Yucatán (15), incluyendo Caulerpella ambigua, resalta la importancia del área de estudio (particularmente la porción caribeña de la península de Yucatán), al ser considerado como uno de los tres centros mundiales con alta diversidad de especies de $\mathrm{Cau}$ lerpa (Prud'Homme et al., 1996). Por ejemplo, el número de especies de Caulerpa en la península de Yucatán (14) es similar al reportado por Price (2011) para las zonas tropical y subtropical del norte de Australia (16). Prud'Homme et al. (1996) mencionaron que el Caribe albergaba 22 especies de Caulerpa, incluyendo siete endémicas; este número de especies es similar al registrado actualmente para el Gran Caribe (comprendiendo el Golfo de México, el Mar Caribe, Bermuda y el litoral norteño de Brasil; Salazar-Vallejo 2000) y aceptadas taxonómicamente en Algaebase, que es de 25 especies, siendo cuatro de ellas endémicas $(C$. floridana W.R. Taylor, $C$. kempfii A.B.Joly et S.M.B.Pereira, $C$. murrayi Weber-van Bosse y C. pusilla (Kützing) J.Agardh; Guiry y Guiry, 2014). No obstante, cabe mencionar que ninguno de estos cuatro taxones endémicos del Gran Caribe ha sido registrado en la península de Yucatán hasta la fecha.

La gran cantidad de variedades (11) y formas (10) de Caulerpa registrados para la península de Yucatán, resalta las dificultades taxonómicas potenciales originadas por la gran plasticidad morfológica existente dentro del género (Carruthers et al., 1993; Silva et al., 1996). Por ejemplo, en el trabajo de Taylor (1969) se evidenciaron los problemas taxonómicos infraespecíficos relacionados con Caulerpa cupressoides: una vez que él había identificado varios especímenes como $C$. cupressoides var. cupressoides, mencionó que eran muy similares a otras cinco variedades. Debido a que una variabilidad morfológica similar ocurrió con $C$. paspaloides, Steidinger y van-Breedveld (1971) decidieron mantener cuatro posibles variedades al nivel de especie. Estos ejemplos enfatizan la necesidad de emprender una revisión taxonómica de Caulerpa en la región de estudio.

Las especies que sobrevivieron a los valores mínimos y en condiciones extremas de salinidad en la península de $\mathrm{Yu}-$ catán fueron $C$. fastigiata (12 PSU, en cenotes, este estudio) y C. prolifera (60 PSU, en Ría Lagartos, Yucatán; OrtegónAznar, 1997). Estos valores de salinidad corresponden a los valores mínimo (13 PSU) y máximo (60 PSU) tolerados por C. lagara en Perth, Australia (Kendrick et al., 1990) y C. lentillifera en Shark Bay, Australia (Carruthers et al., 1993), respectivamente.

El análisis de aglomeración, con los datos de presenciaausencia de las Caulerpaceae, no mostró algún arreglo definido en lo que respecta a los sitios de recolecta para ambos lados de la península de Yucatán. En contraste, los estudios de Aguilar-Rosas et al. (2001), con taxones de Phaeophyceae, y Díaz-Martín y Espinoza-Avalos (2000), con taxones de Halimedaceae y Udoteaceae, proponen que Cabo Catoche es el punto geográfico costero que separa los elementos ficoflorísticos de ambos lados de la península i.e., Golfo de México y Caribe mexicano. La punta noreste de la península igualmente se ha sugerido por otros autores para separar subprovincias (Salazar-Vallejo, 2000), regiones (Costello et al., 2010) y ecoregiones (Spalding et al., 2007) zoogeográficas. Díaz-Martín y Espinoza-Avalos (2000) registraron que el $21 \%$ de 63 especies Phaeophyceae no se compartían en ambos lados de la península, mientras que Aguilar-Rosas $e t$ al. (2001) reportaron que el $68 \%$ de 41 taxones de Halimedaceae y Udoteaceae registrados en el Caribe mexicano no se había registrado en el lado del Golfo de México. En los dos trabajos anteriores se dan elementos diferenciales existentes hacia ambos lados de la península, que podrían explicar las diferencias florísticas que ellos registraron, como la dimensión de la plataforma continental, los tipos de sustrato, las velocidades de corriente, la presencia de surgencias y la extensión de comunidades de arrecifes de coral. Sin embargo, esas mismas características diferenciales no fueron suficientes para separar los sitios por la presencia de taxones Caulerpaceae. Por otro lado, no se puede descartar que un muestreo cuantitativo (estimando abundancia y biomasa de ejemplares, por ejemplo) y más equitativo en el número de sitios de colecta con presencia de taxones Caulerpaceae (menor en el lado del Golfo de México que en el lado caribeño; Tabla 2), pudiera revelar una agrupación distintiva de sitios a ambos lados de la península.

Cabe señalar el caso particular de Caulerpa brachypus $\mathrm{f}$. parvifolia, especie nativa del océano Pacífico y considerada como un alga invasora en las aguas del sureste de Florida 
donde se registró por primera vez en mayo de 2001 (Lapointe et al., 2005). Este taxón invasivo también ha sido referido como C. brachypus Harvey (Trowbridge, 2004; Walters et al., 2006; Glardon et al., 2008). Sin embargo, cuando los autores (Jacoby et al., 2004; Banks et al., 2005) presentaron fotografías es claro que la especie invasora pertenece a $C$. brachypus f. parvifolia, y lo mismo sería para los registros de las Antillas Mayores y Antillas Menores dados por Littler y Littler (2000). En comparación a la forma parvifolia, C. brachypus Harvey var. brachypus tiene fronda ligulada, alcanza 5-6 (-9) cm de largo, frecuentemente (sub-)dicotómica o con proliferaciones en el ápice, con márgenes irregularmente constrictos y algunos con espinas mucromadas (Coppejans y Prud'Homme van Reine, 1992).

Además de Australia, la forma parvifolia se ha registrado en China, Filipinas, Indonesia, Japón, Nueva Zelanda, Fiyi y África (Silva et al., 1996; Guiry y Guiry, 2014), por lo que cada una de esas zonas es una fuente potencial del alga invasora. El sitio y modo de introducción de $C$. brachypus f. parvifolia al Atlántico occidental/Caribe se desconoce. Algas pertenecientes a este taxón se han reportado de Florida, Antillas Mayores, Antillas Menores y Quintana Roo, México. Acuaristas profesionales y aficionados pudieran ser los vectores de la introducción de $C$. brachypus f. parvifolia. Por ejemplo, Walters et al. (2006) obtuvieron Caulerpa spp., de 30 páginas de internet, que representaron 25 estados de EUA y Gran Bretaña.

Caulerpa brachypus f. parvifolia desapareció de las aguas enfrente de Xcaret después del paso de los huracanes Emily y Wilma en 2005. Del mismo modo, las algas de este taxón fueron completamente removidas por el paso de los huracanes Frances, Jeanne (en 2004) y Wilma (in 2005) por el área de Florida (Lapointe et al., 2006; Lapointe y Bedford, 2010). Es importante resaltar que tapetes de esta alga invasora creciendo en los tanques de exhibición de Xcaret sobrevivieron por un año más después de su desaparición en el mar.

En resumen, en la península de Yucatán se ha registrado el $60 \%$ de especies de Caulerpaceae que habita en la cuenca del Mar Caribe, uno de los centros mundiales de alta diversidad de especies de esta familia de macroalgas. Se registra por primera vez a la especie invasora Caulerpa brachypus f. parvifolia, taxón que coloniza agresivamente sustratos y que desplaza flora y fauna nativa de ambientes arrecifales (Jacoby et al., 2004; Lapointe et al., 2005), similares a los que se encuentran principalmente en el lado caribeño de la Península. Las especies de Caulerpaceae no se agruparon diferencialmente en ambos lados de la Península, el caribeño y del Golfo de México, como se ha reportado para otros grupos de organismos marinos.

\section{Agradecimientos}

Agradecemos a M. D. Esquivel-Moreno, por su apoyo en la colecta de campo; E. Torres-Mejía y M. A. A. Díaz-Mar- tín, por su ayuda en el campo y por separar las Caulerpaceae de muestras de algas y pastos marinos; A. Ortega-Jasso, por proporcionar los especímenes y datos de cenotes y caletas; J. J. Bolaños-Guerra, J. A. Bautista-Martínez y A. Naal-Gutiérrez, por su apoyo para recolectar C. brachypus f. parvifolia; N. P. Cetz-Navarro, por elaborar las figuras 1 y 2 , y sus comentarios al manuscrito; y a C. G. MontañoMoctezuma, por su orientación con los análisis estadísticos y sus valiosos comentarios al manuscrito. Dos revisores anónimos ayudaron a mejorar sustancialmente la versión final del manuscrito.

\section{Literatura citada}

Aguilar-Rosas M.A., Aguilar-Rosas L.E. y Aguilar-Rosas R. 1998. Algas marinas de la región central de Quintana Roo, México. Polibotánica 7:15-32.

Aguilar-Rosas M.A., Aguilar-Rosas L.E. y Fernández-Prieto J.A. 1989. Algas marinas bentónicas de la bahía de la Ascención, Quintana Roo, México. Boletín del Instituto de Oceanografía de Venezuela 28:67-75.

Aguilar-Rosas L.E., Aguilar-Rosas M.A., Gómez-Pedroso-Cedillo A. y Fernández-Prieto J.A. 1992. Adiciones a la flora marina del caribe mexicano. Acta Botanica Mexicana 19:77-84.

Aguilar-Rosas L.E., Espinoza-Avalos J. y Aguilar-Rosas R. 2001. Distribución de las especies de la familia Udoteaceae (Bryopsidales, Chlorophyta) de la península de Yucatán, México. Anales de la Escuela Nacional de Ciencias Biológicas 47:99-108.

Apartado F.P., Dreckmann K.M. y Sentíes-Granados A. 2002. Caulerpa mexicana Sonder ex Kutzing (Caulerpaceae, Chlorophya) en México. Polibotánica 13:97-105.

Banks K., Beaver C., Bohnsack J., Dodge R.E., Gilliam D., Jaap W., Keller B., Leeworthy V.R., Matthews T., Ruiz-Carus R., Santavy D. y Spieler R. 2005. The state of coral reef ecosystems of Florida. En: Waddell J.E. Ed. The State of Coral Reef Ecosystems of the United States and Pacific Freely Associated States: 2005, pp. 150-200. Silver Spring, Maryland.

Bastida-Zavala J.R., Beltrán-Torres A.U., Gutierrez-Aguirre M.A. y Fuente-Betancourt G. 2000. Rapid assessment of reef patches in Majagual, Quintana Roo, Mexico. Revista de Biología Tropical 48:137-143.

Callejas-Jiménez M.E., Sentíes-Granados A. y Dreckmann K.M. 2005. Macroalgas bentónicas de Puerto Real, Faro Santa Rosalía y Playa Preciosa, Campeche, México, con algunas consideraciones florísticas y ecológicas para el estado. Hidrobiológica 15:89-96.

Carruthers T.J.B., Walker D.I. y Huisman M. 1993. Culture studies on two morphological types of Caulerpa (Chlorophyta) from Perth, Western Australia, with a description of a new species. Botanica Marina 36:589-596.

Clarke K.R. y Gorley R.N. 2006. PRIMER v6: User manual / Tutorial. PRIMER-E, Plymouth.

Collado-Vides L., González-González J. y Ezcurra E. 1995. Patrones de distribución ficoflorística en el sistema lagunar de Nichupté, Quintana Roo, México. Acta Botanica Mexicana 31:19-32.

Collado-Vides L., Ortegón-Aznar I., Sentíes-Granados A., CombaBarrera L. y González-González J. 1998. Macroalgae of Puer- 
to Morelos reef system, Mexican Caribbean. Hidrobiológica 8:133-143.

Coppejans E. y Prud'homme van Reine W.F. 1992. Seaweeds of the Snellius-II Expedition (E. Indonesia): the genus Caulerpa (Chlorophyta-Caulerpales). Bulletin Séances Academie Royale des Sciences D'Outre-Mer - Mededelingen del Zittingen Koninklijke Academie vor Overzeese Wetenschappen 37:667-712

Costello M.J., Coll M., Danovaro R., Halpin P., Ojaveer H. y Miloslavich P. 2010. A census of marine biodiversity knowledge, resources, and future challenges. PLoS ONE 5:e12110.

Dawes C.J. y Mathieson A.C. 2008. The Seaweeds of Florida. University Press of Florida, Gainesville.

De-Rios N.R. 1972. Contribución al estudio sistemático de las algas macroscópicas de las costas de Venezuela. Acta Botánica Venezuélica 7:219-324.

Díaz-Martín M.A. y Espinoza-Avalos J. 2000. Distribution of brown seaweeds (Phaeophyta) in the Yucatan peninsula, Mexico. Bulletin of Marine Science 66:279-289.

Díaz-Martín M.A. y Quan-Young L.I. 2001. Ampliación de ámbito de 32 macroalgas de Isla Mujeres, Quintana Roo, México. Revista de Biología Tropical 49:391-394.

Díaz-Martin M.A., Torres-Mejía E. y Espinoza-Avalos J. 1998. Lista de algas del Área de Protección Yum Balam, Quintana Roo, México. Revista de Biología Tropical 46:487-492.

Díaz-Merlano J.M. y Puyana-Hegedus M. 1994. Moluscos del Caribe Colombiano. Un Catálogo Ilustrado. Colciencias, Fundación Natura e Invemar, Santafé de Bogotá.

Espinoza-Avalos J., Hernández-Arana H.A., Álvarez-Legorreta T., Quan-Young L.I., Oliva-Rivera J.J., Valdez-Hernández M., Zavala-Mendoza A., Cruz-Piñón G., López C.Y., SepúlvedaLozada A., Worum-Ference P., Villegas-Castillo A. y van Tussenbroek B.I. 2009. Vegetación acuática sumergida. En: Espinoza-Avalos J., Islebe G.A. y Hernández-Arana H.A. Eds. El Sistema Ecológico de la Bahía de Chetumal / Corozal: Costa Occidental del Mar Caribe, pp. 28-40, El Colegio de la Frontera Sur, Chetumal.

Freile-Pelegrín Y. y Morales J.L. 2004. Antibacterial activity in marine algae from the coast of Yucatan, Mexico. Botanica Marina 47:140-146.

Garduño-Solórzano G., Godínez-Ortega J.L. y Ortega M.M. 2005. Distribución geográfica y afinidad por el sustrato de las algas verdes (Chlorophyceae) bénticas de las costas mexicanas del Golfo de México y Mar Caribe. Boletín de la Sociedad Botánica de México 76:61-78.

Garza-Barrientos M.A. 1975. Primeras consideraciones referentes sobre la flora marina del sureste de la república mexicana. $M e$ morias de Simposio Latinoamericano de Oceanografía Biológica, Universidad de Oriente 2:7-27.

Glardon C.G., Walters L.J., Quintana-Ascencio P.F., McCauley L.A., Stam W.T. y Olsen J.L. 2008. Predicting risks of invasion of macroalgae in the genus Caulerpa in Florida. Biological Invasions 10:1147-1157.

Guiry M.D. y Guiry G.M. 2014. AlgaeBase. World-wide electronic publication, National University of Ireland, Galway. <http:// www.algaebase.org > (consultado 8 abril 2014).

Huerta-Múzquiz L. 1958. Contribución al conocimiento de las algas de los bajos de la Sonda de Campeche, Cozumel e Isla Mujeres. Anales de la Escuela Nacional de Ciencias Biológicas, México 9:115-123.

Huerta-Múzquiz L. y Garza-Barrientos M.A. 1966. Algas marinas del litoral del estado de Campeche. Ciencia 24:193-200.

Huerta-Múzquiz L., Mendoza-González A.C. y Mateo-Cid L.E. 1987. Avance sobre un estudio de las algas marinas de la península de Yucatán. Phytologia 62:23-53.

Humm H.J. 1952. Marine algae from Campeche banks. Florida State University Studies 7:27.

Jacoby C., Lapointe B. y Creswell L. 2004. Are native and nonindigenous seaweeds overgrowing Florida's East coast reefs? Florida Sea Grant College Program SGEF-156. Disponible en: $<$ https://www.flseagrant.org/wp-content/uploads/flsgpg01015. pdf>

John D.M. y Price J.H. 1979. The marine benthos of Antigua (Lesser Antilles) I. Environment, distribution and ecology. Botanica Marina 22:313 -326.

Kendrick G.A., Huisman J.M. y Walker D.I. 1990. Benthic macroalgae of Shark Bay, Western Australia. Botanica Marina 33:47-54.

Lapointe B.E., Barile P.J., Littler M.M. y Littler D.S. 2005. Macroalgal blooms on southeast Florida coral reefs: II. Cross-shelf discrimination of nitrogen sources indicates widespread assimilation of sewage nitrogen. Harmful Algae 4:1106-1122.

Lapointe B.E. y Bedford B.J. 2010. Ecology and nutrition of invasive Caulerpa brachypus f. parvifolia blooms on coral reefs off southeast Florida, U.S.A. Harmful Algae 9:1-12.

Lapointe B.E., Bedford B.J. y Baumberger R. 2006. Hurricanes Frances and Jeanne remove blooms of the invasive green alga Caulerpa brachypus forma parvifolia (Harvey) Cribb from coral reefs off northern Palm Beach County, Florida. Estuaries and Coasts 29:966-971.

Littler D.S. y Littler M.M. 2000. Caribbean Reef Plants: an Identification Guide to the Reef Plants of the Caribbean, Bahamas, Florida and Gulf of Mexico. OffShore Graphics, Inc., Washington. D.C.

Mateo-Cid L.E., Mendoza-González A.C. y Searles R.B. 2006. A checklist and seasonal account of the deepwater Rhodophyta around Cozumel island on the Caribbean coast of Mexico. $\mathrm{Ca}$ ribbean Journal of Science 42:39-52.

Mendoza-González A.C. y Mateo-Cid L.E. 1992. Algas marinas bentónicas de Isla Mujeres, Quintana Roo, México. Acta Botanica Mexicana 19:37-61.

Mendoza-González A.C., Mateo-Cid L.E. y Searles R.B. 2000. New records of benthic marine algae from Isla Cozumel, Mexico: Phaeophyta and Chlorophyta. Bulletin of Marine Science 66:119-130.

Mendoza-González A.C., Mateo-Cid L.E. y Searles R.B. 2007. Yucatan seaweeds from the offshore waters of Isla Mujeres, Quintana Roo, Mexico. Botanica Marina 50:280-287.

Norris J.N. y Bucher K.E. 1982. Marine algae and seagrasses from Carrie Bow Cay, Belize. Smithsonian Contribution Marine Science 12: 167-223.

Okolodkov Y.B., Merino-Virgilio F.C., Aké-Castillo J.A., AguilarTrujillo A.C., Espinosa-Matías S. y Herrera-Silveira J.A. 2014. Seasonal changes in epiphytic dinoflagellate assemblages near the northern coast of the Yucatan Peninsula, Gulf of Mexico. Acta Botanica Mexicana 107:121-151.

Ortega M.M. 1995. Observaciones del fitobentos de la Laguna de Términos, Campeche, México. Anales Instituto Biología UNAM, México, Serie Botánica 66:1-36.

Ortegón-Aznar I. 1997. Estudio de integración ficoflorística de tres lagunas costeras de la península de Yucatán. Tesis de Maes- 
tría, Facultad de Ciencias, Universidad Nacional Autónoma de México, D.F. 143 pp.

Ortegón-Aznar I., González-González J. y Sentíes-Granados A. 2001. Estudio ficoflorístico de la laguna de Río Lagartos, Yucatán, México. Hidrobiológica 11:97-104.

Ortegón-Aznar I., Leon-Tejera H., Gold-Morgan M. y RamírezMiss N. 2008. Preliminary results on marine algae of Madagascar Reef, Yucatan, México: a functional group approach. Proceedings of the 11th International Coral Reef Symposium, pp. 1373-1376. Ft. Lauderdale, Florida, 7-11 july.

Ortegón-Aznar I., Sánchez-Molina I. y Casanova-Cetz R.H. 2009. The distribution of marine algae in a coastal lagoon, northern Yucatan Peninsula, Mexico. Neotropical Biology and Conservation 42:99-105.

Pacheco-Cervera M.C., Pacheco-Ruíz I., Ramos-Miranda J., Cetz-Navarro N.P. y Soto-Ávila J.L. 2010. Presencia del género Caulerpa en la Bahía de Campeche, Camp. Hidrobiológica 20:57-69.

Price I.R. 2011. A taxonomic revision of the marine green algal genera Caulerpa and Caulerpella (Chlorophyta, Caulerpaceae) in northern (tropical and subtropical) Australia. Australian Systematic Botany 24:137-213.

Price I.R., Huisman J.M. y Borowitzka M.A. 1998. Two new species of Caulerpa (Caulerpales, Chlorophyta) from the west coast of Australia. Phycologia 37:10-15.

Prud'homme Van Reine W., Verheij E. y Coppejans E. 1996. Species and ecads of Caulerpa (Ulvophyceae, Chlorophyta) in Malesia (South-East Asia): Taxonomy, biogeography and biodiversity. Netherlands Journal of Aquatic Ecology 30:83-98.

Quan-Young L.I., Díaz-Martín M.A. y Espinoza-Avalos J. 1998. Caulerpa webbiana (Chlorophyta: Caulerpaceae) en la península de Yucatán, México. Revista de Biología Tropical 46:847.

Quan-Young L.I., Díaz-Martin M.A. y Espinoza-Avalos J. 2004. Floristics, cover, and phenology of marine macroalgae from Bajo Pepito, Isla Mujeres, Mexican Caribbean. Bulletin of Marine Science 75:11-25.

Quan-Young L.I., Díaz-Martín M.A. y Espinoza-Avalos J. 2006. Algas epífitas de Bajo Pepito, Isla Mujeres, Quintana Roo, México. Revista de Biología Tropical 54:317-328.

Robledo D. y Freile-Pelegrín Y. 2005. Seasonal variation in photosynthesis and biochemical composition of Caulerpa spp. (Bryopsidales, Chlorophyta) from the Gulf of Mexico. Phycologia 44:312-319.

Recibido: 13 de junio de 2014

Aceptado: 10 de octubre de 2014
Salazar-Vallejo S.I. 2000. Biogeografía marina del Gran Caribe. Interciencia 25:7-12.

Serviere-Zaragoza E., Collado-Vides L. y González-González J. 1992. Caracterización ficológica de la Laguna de Bojórquez, Quintana Roo, México. Caribbean Journal of Science 28:126-133.

Silva P.C., Basson P.W. y Moe R.L. 1996. Catalogue of the Benthic Marine Algae of the Indian Ocean. University of California Publications in Botany vol. 79, University of California Press, Estados Unidos de America.

Spalding M.D., Fox H.E, Allen G.R., Davidson N., Ferdaña Z.A., Finlayson M., Halpern B.S, Jorge M.A., Lombana A., Lourie S.A., Martin K.D., McManus E., Molnar J., Recchia C.A. y Robertson J. 2007. Marine ecoregions of the world: A bioregionalization of coastal and shelf areas. Bioscience 57:573-583.

Steidinger K.A. y Van Breedveld J.F. 1971. Benthic Marine Algae from Waters Adjacent to the Crystal River Electric Power Plant (1969 and 1970). Professional Papers Series No. 16., Florida Department of Natural Resources, Marine Research Laboratory, St. Petersburg.

Taylor W.R. 1960. Marine Algae of the Eastern Tropical and Subtropical Coast of the Americas. University Michigan Press, Ann Arbor.

Taylor W.R. 1969. Notes on the distribution of West Indian marine algae particularly in the Lesser Antilles. Contribution University of Michigan Herbarium 9:125-203.

Trowbridge C.D. 2004. Emerging associations on marine rocky shores: specialist herbivores on introduced macroalgae. Journal of Animal Ecology 73:294-308.

van Tussenbroek B.I. y Collado-Vides L. 2000. Filamentous algae dominate a tropical reef community in the Mexican Caribbean: an unexpected organization of reef vegetation. Botanica Marina 43:547-557.

Walters L.J., Brown K.R., Stam W.T. y Olsen J.L. 2006. E-commerce and Caulerpa: unregulated dispersal of invasive species. Frontiers in Ecology and the Environment 4:75-79.

Weber-van-Bosse A. 1898. Monographie des Caulerpes. Annales du Jardin Botanique de Buitenzorg 15:243-401.

Wynne M.J. 2011. A checklist of benthic marine algae of the tropical and subtropical Western Atlantic: third revision. Nova Hedwigia Beihefte 140, J. Cramer, Stuttgart.

Zubia M., Robledo D. y Freile-Pelegrín Y. 2007. Antioxidant activities in tropical marine macroalgae from the Yucatan Peninsula, Mexico. Journal of Applied Phycology 19:449-458. 